\title{
Relação entre variáveis psicossociais familiares e desempenho em leitura/escrita em crianças
}

\author{
Jacqueline Raquel Bianchi Enricone \\ Jerusa Fumagalli de Salles
}

\begin{abstract}
Resumo
Este artigo analisa a relação entre fatores psicossociais familiares e o desempenho em leitura e escrita a partir de um estudo comparativo realizado através de entrevista semiestruturada com 29 familiares de dois grupos de alunos de $2^{\text {a }}$ série do ensino fundamental. Por meio de avaliação neuropsicológica, os estudantes foram caracterizados em: com dificuldade de leitura e escrita e leitores e escritores competentes. As análises estatísticas evidenciaram associação significativa entre o grupo com dificuldade em leitura e escrita e as variáveis presença de transporte próprio na família, percepção dos familiares de que a criança teve dificuldades para aprender a ler e no desempenho da leitura, maior índice de repetência escolar e história familiar de dificuldade na leitura. Os resultados demonstram a importância da análise de fatores psicossociais familiares para a compreensão da complexidade que envolve o desenvolvimento de habilidades de leitura e escrita em crianças. Palavras-chave: Dificuldades escolares, família, neuropsicologia.
\end{abstract}

\section{Relationship between family psychosocial variables and reading/writing performance in children}

\begin{abstract}
In this article we analyze the relationship between family psychosocial factors and the reading and writing performance in children. We conducted a comparative study through a semi-structured interview with 29 families of two groups of second grade students from elementary education. After the neuropsychological assessment, the students were characterized like either having trouble reading and writing or being efficient readers and writers. The statistic analysis highlighted the significant association between the group with troubles in reading and writing and the variables, like the presence of their own family transportation, the family's perception on the child's trouble learning to read, as well as his/her difficulty in reading performance, the children's higher index of repeating the school year and the family's history of difficulties in reading. The results obtained showed the importance of analyzing family psychosocial factors in order to understand the complex process of developing children's reading and writing skills.
\end{abstract}

Key words: School difficulties, family, neuropsychology.

\section{Relación entre variables psicosociais familiares y rendimiento de niños en lectura/escritura}

\section{Resumen}

Este artículo analiza la relación entre factores psicosociales familiares y el rendimiento en lectura y escritura a partir de estudio comparativo realizado a través de entrevista semi-estructurada con 29 familiares de dos grupos de alumnos de 20 año de enseñanza básica. Por medio de evaluación neuropsicológica, se caracterizó a los estudiantes en: "con dificultad de lectura y escritura" y "lectores y escritores competentes". Los análisis estadísticos mostraron asociación significativa entre el grupo con dificultad en lectura y escritura y las variables presencia de transporte propio en la familia, percepción de los familiares de que el niño tuvo dificultades para aprender a leer y en el rendimiento de la lectura, mayores índices de repetición escolar e historia familiar de dificultad en lectura. Los resultados demuestran la importancia del análisis de factores psicosociales familiares para la comprensión de la complejidad que envuelve el desarrollo de habilidades de lectura y escritura en niños.

Palabras Clave: Dificultades escolares, familia, neuropsicología. 


\section{Introdução}

A leitura e a escrita são atividades complexas que envolvem muitos processos. No Brasil, as estatísticas governamentais apontam que as dificuldades de leitura e escrita persistem como um problema educacional que não está superado. Os resultados do Pisa (Programa Internacional de Avaliação de Alunos), em 2006, divulgados pela OCDE (Organização para a Cooperação e o Desenvolvimento Econômico), mostram que os alunos brasileiros obtiveram médias que os colocam na $48^{a}$ posição em leitura, entre 56 países. Em 2007, pesquisa do IBGE apontou que, entre as 28,3 milhões de crianças de 7 a 14 anos, que, pela idade, já teriam passado pelo processo de alfabetização, 2,4 milhões $(8,4 \%)$ não sabiam ler e escrever, sendo que $87,2 \%$ delas frequentavam estabelecimentos de ensino (IBGE, 2008).

As dificuldades escolares têm inquietado diferentes pesquisadores preocupados com a educação e remetem a debates sobre a aprendizagem, sobre a eficácia dos professores, sobre o serviço público, sobre o montante de recursos que o país deve investir no seu sistema educacional, sobre os modos de vida e o trabalho na sociedade, sobre igualdade de oportunidades, sobre cidadania etc. (Charlot, 2000, p.14). Os dados estatísticos, somados à complexidade do processo educativo, demonstram a importância da continuidade de estudos que contribuam para a compreensão de tal fenômeno sobre diferentes perspectivas. Dessa forma, este artigo privilegia as variáveis psicossociais familiares como foco de estudo relacionado ao desempenho em leitura e escrita em crianças.

No DSM IV-TR (2002), a dificuldade em leitura é descrita como Transtorno Específico de Leitura, envolvendo um rendimento (correção, velocidade, compreensão da leitura) abaixo do esperado para a idade cronológica, a inteligência medida e a escolaridade do indivíduo, que interfere no desempenho escolar e em atividades cotidianas que exigem habilidade leitora. Da mesma forma, o Transtorno da Expressão Escrita envolve habilidades de escrita acentuadamente abaixo do nível esperado, considerando as mesmas variáveis.

Com relação à caracterização das dificuldades na leitura, tomando como referência os modelos de dupla rota ou múltiplas rotas de leitura e de escrita (Ellis, 1995; Ellis \& Young, 1988; Hillis \& Caramazza, 1992), é possível descrever três tipos de dislexias de desenvolvimento: a fonológica, que se caracteriza por uma incapacidade de decodificação fonológica grave (rota fonológica); a dislexia de superfície, que envolve uma incapacidade no nível do tratamento ortográfico da informação (rota lexical); e a dislexia mista (fonológica e lexical), que é caracterizada pela presença de distúrbios de decodificação fonológica e no processo ortográfico ao mesmo tempo.

$\mathrm{Na}$ abordagem cognitiva, a leitura envolve o reconhecimento de palavras e a compreensão da linguagem. $O$ que distingue o leitor fluente do leitor iniciante ou com dificuldades é que, enquanto estes se esforçam para identificar as palavras de um texto, o leitor fluente reconhece-as com rapidez, de memória, e pode concentrar sua atenção no significado do texto (Cardoso-Martins, 2008).

Com relação à etiologia, Frith (1997) afirma a possibilidade de uma predisposição cerebral para a dislexia, gerada por condições biológicas em interação com o ambiente, que pode conduzir à disfunção em um ou mais componentes mentais envolvidos na habilidade de ler e escrever, como o processamento fonológico, ou seja, o uso da estrutura fonológica ou sonora da linguagem oral. Em termos cognitivo-linguísticos, há déficits no processamento fonológico da linguagem, como consciência fonológica, memória de curto prazo verbal e lentidão de acesso à informação fonológica na memória de longo prazo (Beitchman \& Young, 1997; Jong, 1998; Morais, 1996; Pennington, 1997; Mayringer \& Wimmer, 2000; Torgesen, Wagner, \& Rashotte, 1994; Winner, 1993).

A amostra de crianças do presente estudo foi analisada em relação a outras habilidades neuropsicológicas e aos subperfis de leitura e escrita (Salles \& Parente, 2006a, 2006b, 2008). Foi encontrada defasagem em consciência fonológica, linguagem oral e memória fonológica das crianças de segunda série com dificuldades de leitura e escrita, quando comparadas a crianças de mesma série, porém sem dificuldades de leitura e escrita. Além disso, as crianças com dificuldade apresentavam desempenho semelhante, nessas habilidades, ao de um grupo mais jovem, de primeira série, emparelhado aos primeiros pelos escores em leitura e escrita de palavras (Salles, 2005; Salles \& Parente, 2006b). $O$ resultado sugere um atraso no desenvolvimento das habilidades de consciência fonológica, memória fonológica e linguagem oral. Nesta amostra com dificuldades de leitura e escrita, também foram encontrados vários subtipos de dificuldades de linguagem escrita e variação dos fatores neuropsicológicos relacionados a cada um deles (Salles \& Parente, 2008). Esses dados apontam que, além dos fatores neurobiológicos e cognitivo-linguísticos, a investigação de fatores psicossociais, relativos ao letramento familiar e ao processo de escolarização, podem ser importantes para melhor compreender a etiologia das dificuldades de leitura e escrita e qualificar a intervenção com essas crianças.

As funções neuropsicológicas, incluindo leitura e escrita, desenvolvem-se na interdependência de vários fatores, por meio de uma interação dinâmica e contínua das experiências sociais e ambientais. Nessa perspectiva, a neuropsicologia transcultural define como seu objeto de estudo mais relevante a influência dos aspectos socioculturais sobre o desenvolvimento cognitivo (Andrade \& Bueno, 2006). Nessa abordagem, o cérebro não funciona como uma variável independente que dita ou controla o comportamento, mas que atua como uma variável dependente, que reflete e é influenciado por fatores ambientais. É a totalidade de ideias, habilidades e costumes em que cada indivíduo nasce e cresce que modula seu desenvolvimento e perfil de habilidades cognitivas (Miranda \& Muszkat, 2004). Pode-se pensar, então, que diferentes ambientes socioculturais influenciam o desenvolvimento de diferentes perfis e habilidades. 
Sendo assim, pode-se afirmar que a aprendizagem e, consequentemente, as dificuldades de aprendizagem da leitura e da escrita sofrem interferência de fatores neuropsicológicos e psicossociais. Dentre os fatores psicossociais, a influência do ambiente familiar no aprendizado escolar é amplamente reconhecida. Fatores como a organização do ambiente físico, a disponibilidade de materiais educacionais, o envolvimento dos pais no processo de desenvolvimento dos filhos, a interação pais-filhos e uso da linguagem no lar, o clima emocional e as práticas educativas utilizadas pela família podem influenciar os processos de aprendizagem escolar das crianças (Marturano, 2000).

O nível socioeconômico, por exemplo, que se refere a uma constelação de fatores, como escolaridade, condições nutricionais, quantidade e qualidade de estimulação, cuidados médicos, riscos perinatais, ocupação, estilos de interação familiar e social e condições de habitação, manifesta-se na integridade do sistema nervoso central e no desempenho neuropsicológico. Assim, o sintoma neuropsicológico precisa estar inserido na circularidade de outros sistemas: emocional, familiar e psicossocial (Miranda \& Muszkat, 2004).

Considerando o exposto, o presente estudo investiga a relação entre fatores psicossociais familiares e o desempenho em leitura e escrita de crianças de $2^{a}$ série com dificuldade de leitura e escrita, comparadas a crianças leitoras e escritoras competentes. Foram analisados, comparativamente, os fatores psicossociais familiares (recursos como ambiente físico, materiais educacionais, acompanhamento familiar na aprendizagem e práticas educativas familiares) e a história prévia de desenvolvimento (linguagem, desenvolvimento motor, relações interpessoais, história de vida escolar) desses dois grupos de crianças e sua possível relação com as habilidades de leitura e escrita.

\section{Método}

\section{Participantes}

A amostra foi composta por vinte e nove familiares de crianças de $2^{a}$ série do ensino fundamental, divididos em dois grupos. O primeiro grupo de participantes deste estudo foi formado por familiares de quinze crianças de $2^{a}$ série que faziam parte do grupo caracterizado como competente em leitura e escrita, com uma média de idade de 8,21 anos (DP $=0,33$ ). O segundo grupo foi estruturado por familiares de catorze alunos de $2^{a}$ série que faziam parte de um grupo com dificuldade de leitura e escrita, com média de idade de $8,43$ anos ( $D P=0,62)$.

Os familiares participantes da pesquisa foram vinte e três mães, três pais, uma irmã, uma prima e uma avó. A média de idade dos pais das crianças do grupo competente foi 37,18 (DP 10,7), enquanto a média de idade das mães foi 36,36 (DP 8,2). Em média, o número de irmãos das crianças deste grupo foi 2,0 (DP 1,2). No grupo de familiares das crianças com dificuldade de leitura e escrita, a média de idade dos pais foi de 38,54 (DP 5,9) e das mães, 39,08 (DP 7,0 ), e a média do número de irmãos foi de 2,6 (DP 1,1).

Foram excluídas da amostra crianças com histórico de problemas neurológicos adquiridos; portadores de necessidades educativas especiais, em regime de inclusão; e com desempenho no teste de Matrizes Progressivas Coloridas de Raven (Angelini, Alves, Custódio, Duarte, \& Duarte, 1999 ) igual ou inferior a $25 \%$.

\section{Delineamento, instrumentos e procedimentos}

Este estudo caracteriza-se como comparativo de grupos contrastantes (Nachmias \& Nachmias, 1996), comparando fatores psicossociais familiares de crianças de $2^{a}$ série com dificuldades de leitura e escrita e de crianças de mesma série e idade, porém leitores e escritores competentes. De uma amostra de 110 crianças de $2^{a}$ série, de cinco escolas públicas estaduais de áreas urbanas de uma cidade do interior do RS, os dois grupos de crianças (competente e com dificuldade de leitura e escrita) foram selecionados pelo desempenho em leitura e escrita de palavras e pseudopalavras isoladas, avaliação da compreensão da leitura textual e avaliação da produção escrita de história (Salles \& Parente, 2005).

O corpo docente das escolas selecionadas, em sua maioria, era composto por profissionais com formação em magistério e nível superior (Pedagogia, Letras, Geografia, entre outros). A média de idade das professoras foi de 44,4 anos (desvio padrão $=10,89$ ) e a média de tempo de atuação no magistério, de 21,6 anos (desvio padrão = 10,76). As escolas atendiam alunos com nível socioeconômico médio e baixo. Estas ofereciam, em geral, ensino fundamental, médio e Educação de Jovens e Adultos (EJA). Os recursos físicos das escolas eram compostos dos seguintes itens: biblioteca, sala de vídeo, laboratório, sala de informática e auditório, além do espaço externo que diferia de tamanho de acordo com a instituição.

Foram consideradas com dificuldades de leitura e escrita crianças com desempenho menor ou igual a 1 , desvio padrão abaixo da média da classe escolar. O grupo competente foi aquele que apresentou escores acima da média da classe escolar (1 desvio padrão acima da média). Informações detalhadas sobre os instrumentos e os procedimentos de aplicação estão descritos em Salles (2005) e Salles \& Parente (2006b, 2007).

Após a seleção dos dois grupos de crianças, todos os pais foram convidados a participar do estudo, sendo realizada entrevista semiestruturada com um familiar de cada criança. O roteiro da entrevista foi fundamentado no Inventário de Recursos e Adversidades do Ambiente Familiar (Marturano, 2006), instrumento que objetiva identificar, no âmbito da família, recursos promotores de desempenho escolar, em um modelo bioecológico de desenvolvimento. Aspectos referentes a dados demográficos da família, organização do ambiente físico e disponibilidade de materiais educacionais, condições e características de desenvolvi- 
Tabela 1. Frequência e percentual da amostra, por grupo, de crianças competentes e com dificuldade em leitura e escrita, segundo variáveis socioeconômicas familiares.

\begin{tabular}{|c|c|c|c|c|c|}
\hline \multirow{2}{*}{ Variável } & & \multicolumn{2}{|c|}{ Grupo competente } & \multicolumn{2}{|c|}{ Grupo com dificuldades } \\
\hline & & frequência & percentual & frequência & percentual \\
\hline \multirow{3}{*}{ Jornada de trabalho do pai } & integral & 8 & 72,7 & 7 & 63,6 \\
\hline & meio turno & 2 & 18,2 & 3 & 27,3 \\
\hline & atípica** & 1 & 9,1 & 1 & 9,1 \\
\hline \multirow{4}{*}{ Jornada de trabalho da mãe } & integral & 4 & 33,3 & 4 & 33,3 \\
\hline & meio turno & 1 & 8,3 & 3 & 25,0 \\
\hline & não trabalha & 3 & 25,0 & 1 & 8,3 \\
\hline & não se aplica*** & 4 & 33,3 & 4 & 33,3 \\
\hline \multirow{3}{*}{ Renda familiar } & até $R \$ 500,00$ & 5 & 41,7 & 7 & 63,6 \\
\hline & $\begin{array}{l}\text { de } R \$ 500,00 \\
\text { a } R \$ 1.000,00\end{array}$ & 5 & 41,7 & 4 & 36,4 \\
\hline & $\begin{array}{l}\text { de } R \$ 1.000,00 \\
\text { a } R \$ 3.000,00\end{array}$ & 2 & 16,6 & 0 & 0,0 \\
\hline \multirow{4}{*}{$\begin{array}{l}\text { Responsável pelo sustento } \\
\text { familiar }\end{array}$} & pai & 5 & 41,7 & 1 & 8,3 \\
\hline & mãe & 1 & 8,3 & 5 & 41,7 \\
\hline & ambos & 5 & 41,7 & 3 & 25,0 \\
\hline & outros $^{\star * * *}$ & 1 & 8,3 & 3 & 25,0 \\
\hline Possui plano de saúde & & $3^{*}$ & 25,0 & $3^{*}$ & 25,0 \\
\hline Possui transporte próprio & & $7^{*}$ & 58,3 & $1^{*}$ & 8,3 \\
\hline
\end{tabular}

Legenda: * $\mathrm{n}=12$; ** horários não definidos; ${ }^{* * *}$ trabalho remunerado realizado em casa; **** avós e irmãos mais velhos.

mento das crianças (linguagem, desenvolvimento psicomotor, relações interpessoais), história de vida escolar, forma de acompanhamento familiar da aprendizagem e práticas educativas familiares foram investigados com os familiares dos dois grupos de crianças (competentes e com dificuldade de leitura e escrita).

Os dados foram analisados de forma descritiva e inferencial. Obteve-se o cálculo de frequência e percentual de cada uma das variáveis psicossociais por grupo. Para a comparação entre os fatores psicossociais familiares de cada um dos grupos, foi empregado o Teste Exato de Fisher, considerando o nível de significância de 5\%.

\section{Resultados}

Quanto ao ambiente físico, no grupo de crianças competentes em leitura e escrita, foi possível verificar que $91,7 \%$ delas residem em casa própria, com média de 6,0 cômodos (DP 2,1) e 2,4 dormitórios (DP 0,7), sendo que $58,3 \%$ das crianças dividem o quarto com outros familiares.
No grupo de crianças com dificuldades na leitura e escrita, verificou-se que $75 \%$ residem em casa própria, com uma média de 5,5 (DP 2,9) cômodos e 2,3 dormitórios (DP 1,0), sendo que $83,3 \%$ das crianças dividem o quarto com outras pessoas da família. A Tabela 1 apresenta as principais variáveis referentes à situação socioeconômica familiar, por grupo.

Observa-se que, no grupo de crianças competentes, há mais mães que não trabalham e maior número de famílias com renda um pouco mais elevada, incluindo transporte próprio. No grupo de crianças com dificuldade, o sustento da casa depende mais da mãe ou de outras pessoas, com uma participação menor do pai. Houve uma associação estatisticamente significativa, no teste exato de Fisher $(p=0,027)$, entre a variável "transporte próprio na família" e grupo de crianças. Mais integrantes do grupo competente em leitura e escrita possuem carro próprio, comparado ao grupo com dificuldades.

A Tabela 2 apresenta os dados referentes à escolaridade e práticas de leitura na família. Com relação à variável escolaridade dos pais, observa-se um menor nível de es- 
colarização geral no grupo de familiares de crianças com dificuldades. No grupo competente, cinco mães e dois pais (7 familiares) têm ensino fundamental incompleto, sendo que, no grupo com dificuldade, este número aumentou para cinco mães e seis pais (11 familiares) que não completaram o ensino fundamental.

Com relação a hábitos de leitura na família, há uma diferença não significativa entre os dois grupos quanto à presença de materiais educativos e relato de hábitos de leitura. Onze familiares do grupo de crianças competentes afirmaram ter hábito de ler revistas ou jornais, sendo que, no grupo de familiares das crianças com dificuldades, nove participantes fizeram a mesma afirmação. Porém, nas famílias das crianças com dificuldades, quatro familiares referiram que a atividade de leitura é esporádica, sendo que a assinatura de revistas e jornais também é menos frequente neste grupo. Em ambos os grupos, houve referência ao hábito de ler histórias infantis; verificou-se, também, que, no grupo competente, quem lê as histórias infantis é a própria criança (citada nove vezes), seguida pela mãe (citada 4 vezes), pai, avós e irmão. Já no grupo com dificuldades, a própria criança foi citada apenas duas vezes, sendo que os familiares envolvidos com a leitura são a mãe (citada oito vezes), o pai (citado três vezes), avó e irmãos.

Houve uma associação estatisticamente significativa, no teste exato de Fisher, entre a variável presença de familiar com história de dificuldade na leitura $(p=0,039)$ e a variável grupo de crianças. Há mais familiares com dificuldade de leitura no grupo com dificuldade em relação ao grupo competente. Entre os familiares com dificuldade de leitura citados no grupo com dificuldade, estão o pai (quatro vezes) e a mãe (cinco vezes); outros familiares citados foram tios (três vezes), primos (duas vezes), avós (duas vezes) e irmão (uma vez). Já no grupo competente, a referência a familiares com dificuldade de aprendizagem na leitura foi mínima, sendo que foram citados a mãe (duas vezes), o pai (uma vez) e a avó (uma vez).

A Tabela 3 apresenta dados referentes ao desenvolvimento infantil das crianças. Não houve associação estatisticamente significativa entre grupos e variáveis referentes ao desenvolvimento infantil. Observa-se uma tendência, sem significância estatística, de que há um maior uso do diálogo como forma de resolução de problemas nos pais do grupo competente.

Tabela 2. Frequência e percentual da amostra, por grupo de familiares de crianças competentes e com dificuldade em leitura e escrita, segundo escolaridade dos pais e práticas de leitura na família.

\begin{tabular}{|c|c|c|c|c|c|}
\hline \multirow{2}{*}{ Variável } & & \multicolumn{2}{|c|}{ Grupo competente } & \multicolumn{2}{|c|}{ Grupo com dificuldade } \\
\hline & & frequência & percentual & frequência & percentual \\
\hline \multirow{2}{*}{ Escolaridade da mãe } & Ensino Fundamental & 6 & 50,0 & 7 & 63,6 \\
\hline & Ensino Médio & 6 & 50,0 & 4 & 36,4 \\
\hline \multirow{2}{*}{ Escolaridade do pai } & Ensino Fundamental & 6 & 54,5 & 9 & 81,8 \\
\hline & Ensino Médio & 5 & 45,5 & 2 & 18,2 \\
\hline $\begin{array}{l}\text { Hábito de leitura de } \\
\text { revistas/jornais }\end{array}$ & & $11^{*}$ & 91,7 & $9^{*}$ & 75,0 \\
\hline Assinatura de revistas/jornais & & $5^{* *}$ & 45,5 & $2^{*}$ & 16,7 \\
\hline $\begin{array}{l}\text { Possui familiar com } \\
\text { dificuldade na leitura }\end{array}$ & & $3^{*}$ & 25,0 & $9^{*}$ & 75,0 \\
\hline \multirow{4}{*}{ Presença de materiais educativos } & jogos & 1 & 8,3 & 1 & 8,3 \\
\hline & jornais & 1 & 8,3 & 0 & 0,0 \\
\hline & computador & 1 & 8,3 & 0 & 0,0 \\
\hline & $\begin{array}{l}\text { livros + jogos + } \\
\text { revistas + jornais }\end{array}$ & 9 & 75,0 & 11 & 91,7 \\
\hline Hábito de ler histórias infantis & & $10^{*}$ & 83,3 & $9^{*}$ & 75,0 \\
\hline \multirow{3}{*}{ Tempo de contato Pai/Criança } & Contato diário & 8 & 66,7 & 6 & 54,5 \\
\hline & Pouco contato & 3 & 25,0 & 2 & 18,2 \\
\hline & Nenhum contato & 1 & 8,3 & 3 & 27,3 \\
\hline \multirow{2}{*}{ Tempo de contato Mãe/Criança } & Contato diário & 11 & 91,7 & 12 & 100,0 \\
\hline & Não se aplica*** & 1 & 8,3 & 0 & 0,0 \\
\hline
\end{tabular}

Legenda: ${ }^{*} n=12 ;{ }^{* *} n=11 ;{ }^{* * *}$ não vive com a mãe. 
Tabela 3.. Frequência e percentual da amostra, por grupo de crianças competentes e com dificuldade em leitura e escrita, segundo variáveis de desenvolvimento infantil e práticas familiares.

\begin{tabular}{|c|c|c|c|c|c|}
\hline \multirow{2}{*}{ Variável } & & \multicolumn{2}{|c|}{ Grupo competente } & \multicolumn{2}{|c|}{ Grupo com dificuldade } \\
\hline & & frequência & percentual & frequência & percentual \\
\hline Houve planejamento familiar & & $2^{* *}$ & 18,2 & $3^{* *}$ & 27,3 \\
\hline Houve problemas na gestação & & $6^{*}$ & 50,0 & $6^{* *}$ & 54,5 \\
\hline Houve problemas no parto & & $3^{* * *}$ & 27,3 & $4^{* *}$ & 36,4 \\
\hline Recém-nascido com problemas & & $3^{* *}$ & 27,3 & $3^{* *}$ & 27,3 \\
\hline Prematuridade & & $3^{*}$ & 25,0 & $0^{*}$ & 0,0 \\
\hline \multirow{2}{*}{ Lateralidade manual } & destro & 10 & 83,8 & 11 & 91,7 \\
\hline & canhoto & 2 & 16,7 & 1 & 8,3 \\
\hline Presença de dificuldade motora & & $1^{* *}$ & 9,0 & $0^{*}$ & 0,0 \\
\hline Pais entendiam a fala & & 9 9* & 81,8 & $9^{*}$ & 75,0 \\
\hline Fala-se outra língua na família & & $1^{*}$ & 8,3 & $0^{*}$ & 0,0 \\
\hline Filho brinca com outras crianças & & $12^{*}$ & 100,0 & $11^{*}$ & 91,7 \\
\hline Pais brincam com a criança & & $9^{*}$ & 75,0 & $12^{*}$ & 100,0 \\
\hline $\begin{array}{l}\text { Houve mudanças familiares nos } \\
\text { últimos } 5 \text { anos }\end{array}$ & & 6 & 50,0 & 9 & 81,8 \\
\hline \multirow{3}{*}{$\begin{array}{l}\text { Forma de resolução de } \\
\text { problemas com o filho }\end{array}$} & diálogo & 5 & 41,7 & 2 & 16,7 \\
\hline & punição & 5 & 41,7 & 3 & 25,0 \\
\hline & ambos & 2 & 16,6 & 7 & 58,3 \\
\hline
\end{tabular}

Legenda: ${ }^{*} n=12 ;{ }^{* *} n=11 ;{ }^{* *} n=10$

Verificou-se, ainda, maior número de mudanças familiares no grupo com dificuldade. Entre as mudanças referidas pelos participantes deste grupo, foram citadas, em maior número, a morte de um familiar (8), a separação dos pais (4), a redução da renda familiar (2), o consumo de álcool ou drogas na família (2), problemas de saúde na família (1), conflitos entre o casal (1) e prisão de familiar (1). As mudanças referidas pelos familiares do grupo competente envolveram a morte de familiar (4), doença na família (3), mudança de cidade (1), doença mental familiar (1), separação dos pais (1), consumo de álcool ou drogas por familiar (1).

Com relação à saúde das crianças do grupo competente, quatro delas faziam algum tipo de tratamento, uma por apresentar alergia, uma com bronquite asmática e duas com problemas hematológicos (anemia). Estas crianças realizavam tratamento médico e apenas um aluno deste grupo fez tratamento psicológico, mas já havia obtido alta. No grupo com dificuldade, os problemas de saúde foram referidos por familiares de cinco crianças e envolviam diagnósticos de bronquite alérgica, sopro cardíaco, anemia, sinusite, alergias e renite alérgica. Uma criança deste grupo fazia tratamento neurológico e utilizava medicação em função de comportamento agitado; uma estava em tratamento psicológico; uma, em tratamento fonoaudiológico; e outra, em acompanhamento fonoaudiológico e psicológico.
Não foi observada discrepância com relação à idade de ingresso na escola entre as crianças de cada grupo. No grupo competente, a idade média de ingresso na primeira série foi de 6,7 anos (DP 0,4) e o ingresso na segunda série foi com 7,7 anos (DP 0,4). No grupo com dificuldade, a média de idade de ingresso na primeira série foi de 6,6 anos (DP 0,4) e a média de ingresso na segunda série foi de 7,7 anos (DP 0,4). Nenhuma criança frequentou classe especial e quatro crianças do grupo com dificuldade têm história de reprovação escolar.

$\mathrm{Na}$ Tabela 4, apresentam-se os dados referentes à vida escolar das crianças na perspectiva dos familiares. Houve associação significativa entre grupos e as variáveis percepção da família sobre dificuldades da criança para aprender a ler $(p=0,012)$ e avaliação do familiar de que a criança tem dificuldade no desempenho da leitura $(p=$ $0,027)$. Portanto, os familiares das crianças com dificuldade na leitura e escrita percebem de forma mais contundente que estas têm dificuldades para aprender a ler e que apresentam dificuldade no desempenho da leitura. Não houve diferença importante com relação à frequência da educação infantil, tempo de contato com a mãe, as mudanças de escola, atividades extraescolares, frequência do familiar na escola e satisfação com a mesma. 
Tabela 4. Frequência e percentual da amostra, por grupo de crianças competentes e com dificuldade em leitura e escrita, segundo variáveis de vida escolar.

\begin{tabular}{|c|c|c|c|c|c|}
\hline \multirow{2}{*}{\multicolumn{2}{|c|}{ Variável }} & \multicolumn{2}{|c|}{ Grupo competente } & \multicolumn{2}{|c|}{ Grupo com dificuldade } \\
\hline & & frequência & percentual & frequência & percentual \\
\hline \multicolumn{2}{|l|}{ Frequentou creche } & $3^{*}$ & 25 & $4^{*}$ & 33,3 \\
\hline \multicolumn{2}{|l|}{ Frequentou pré-escola } & $9^{* *}$ & 81.8 & $10^{*}$ & 83,3 \\
\hline \multicolumn{2}{|l|}{ Mudança de escola } & $6^{*}$ & 50,0 & $6^{*}$ & 50,0 \\
\hline \multirow{2}{*}{$\begin{array}{l}\text { Rendimento escolar } \\
\text { conforme } \\
\text { Familiar }\end{array}$} & Bom/ótimo & 11 & 91,7 & 6 & 50,0 \\
\hline & Regular/Insuficiente & 1 & 8,3 & 6 & 50,0 \\
\hline Desempenho leitura & Bom/ótimo & 11 & 91,7 & 5 & 41,7 \\
\hline conforme familiar & Regular/Insuficiente & 1 & 8,3 & 7 & 58,3 \\
\hline \multicolumn{2}{|l|}{$\begin{array}{l}\text { Teve dificuldade para } \\
\text { aprender a ler }\end{array}$} & $3^{*}$ & 25,0 & $10^{*}$ & 83,3 \\
\hline \multicolumn{2}{|l|}{$\begin{array}{l}\text { Possui atividades } \\
\text { extraescolares }\end{array}$} & $2^{*}$ & 16,7 & $3^{*}$ & 25,0 \\
\hline \multicolumn{2}{|l|}{$\begin{array}{l}\text { Faz tarefas escolares } \\
\text { Sozinho }\end{array}$} & $10^{*}$ & 83,3 & $8^{*}$ & 66,7 \\
\hline \multicolumn{2}{|l|}{$\begin{array}{l}\text { Tem horário para } \\
\text { tarefas escolares }\end{array}$} & $10^{*}$ & 83,3 & $8^{*}$ & 66,7 \\
\hline \multicolumn{2}{|l|}{$\begin{array}{l}\text { Tem horário rígido para } \\
\text { tarefas diárias }\end{array}$} & $11^{*}$ & 91,7 & $8^{*}$ & 66,7 \\
\hline \multicolumn{2}{|l|}{ Tem local para tarefa } & $11^{*}$ & 91,7 & $9^{* *}$ & 81,8 \\
\hline \multicolumn{2}{|l|}{$\begin{array}{l}\text { Familiar satisfeito com } \\
\text { a escola }\end{array}$} & $11^{*}$ & 91,7 & $12^{*}$ & 100,0 \\
\hline \multicolumn{2}{|c|}{$\begin{array}{l}\text { Familiar frequenta escola do } \\
\text { filho }\end{array}$} & $11^{*}$ & 91,7 & $12^{*}$ & 100,0 \\
\hline
\end{tabular}

Legenda: ${ }^{*} \mathrm{n}=12 ;{ }^{* *} \mathrm{n}=11$.

Foi possível, ainda, observar algumas tendências, mesmo que na análise estatística não tenham sido significativas: uma maior observação de rendimento regular ou insuficiente da criança na escola pelos familiares do grupo com dificuldade; mais relatos de que as crianças do grupo competente fazem as tarefas escolares sozinhas, têm mais horários e local definido para as tarefas e têm um pouco mais de tempo de contato com o pai.

\section{Discussão}

A neuropsicologia transcultural propõe ampliar o olhar sobre os fenômenos e tentar compreender o ser humano em seu desenvolvimento do ponto de vista biológico, neurológico, psicológico, histórico, social e cultural. Isso significa romper com a dualidade corpo/mente, razão/emoção, genética/ ambiente e pensar a partir da integração do conhecimento de diferentes áreas. Como foi possível observar, este estudo revelou que algumas variáveis psicossociais familiares estão relacionadas de forma mais significativa com o grupo de crianças com dificuldades de leitura/escrita. Houve associação significativa entre este grupo de crianças e as variáveis presença de transporte próprio na família (mais frequente no grupo competente), percepção dos familiares de que a criança teve dificuldades para aprender a ler, avaliação do familiar de que criança tem dificuldade no desempenho da leitura, repetência escolar e história familiar de dificuldade na leitura (mais frequente no grupo com dificuldade). Como a amostra de cada grupo era pequena, algumas tendências encontradas não atingiram grau de significância estatística.

Quanto aos fatores socioeconômicos familiares, observou-se que, no grupo com dificuldades de leitura e escrita, há maior número de filhos, menor renda e menor número de famílias que residem em casa própria, sendo que mais crianças dividem seu quarto com outros familiares. Neste grupo, o sustento da casa está mais vinculado à figura da mãe ou de outras pessoas, com uma participação menor do pai, sendo que a maior parte das famílias não tem carro próprio. Embora isoladamente estas informações não sejam muito relevantes, a integração de todos esses dados aponta diferença entre os dois grupos, sugerindo que as crianças com dificuldade na leitura e escrita podem apresentar menor nível socioeconômico familiar.

O baixo nível socioeconômico tem sido entendido como fator de risco para o desenvolvimento psicológico e 
social do ser humano. Entre os fatores de risco, estão a baixa remuneração parental, baixa escolaridade, famílias numerosas e ausência de um dos pais (Hutz, Koller, \& Bandeira, 1996). Para Miranda e Muszkat (2004), também o nível socioeconômico afeta o desempenho cognitivo e se manifesta na integridade do sistema nervoso central e no desempenho neuropsicológico. Neste estudo, os fatores de risco citados estão mais presentes, mesmo que de forma não significativa, no grupo de crianças com dificuldades na leitura.

Observou-se menor nível de escolarização nos pais do grupo com dificuldades. Alguns familiares do grupo de crianças com dificuldades informam que a atividade de ler histórias infantis em casa não é frequente, o que denota que estas crianças podem ter menos estímulos familiares e uma menor experiência com a leitura.

O fato de o grupo com dificuldade ter mais familiares com história de dificuldade de leitura pode ser interpretado de duas formas. Pode-se pensar, inicialmente, no modelo etiológico de Frith (1997), o qual, como já visto, afirma que condições biológicas em interação com o ambiente podem ter efeitos adversos no desenvolvimento cerebral, causando distúrbios como a dislexia. Segundo a autora, fatores biológicos, genéticos, em interação com o ambiente, acarretam déficits cognitivos manifestados por prejuízos na leitura e escrita. Então, seria possível uma predisposição genética para a dificuldade na leitura e escrita. Pode-se, também, pensar que estes familiares com dificuldades na leitura, que em maioria são o pai e/ou a mãe, podem não ser leitores fluentes ou assíduos, pois, embora os familiares tenham informado que existem hábitos de leitura em casa, alguns referem que esta atividade não é frequente. Também é importante destacar que as respostas positivas para hábitos de leitura podem estar influenciadas pelo critério do que é socialmente esperado para esta pergunta e que, sendo assim, os entrevistados possam ter hipervalorizado uma atividade que, na verdade, não é comum no cotidiano da família.

Aliando esta visão multifatorial, Aaron (1995), Aaron, Joshi, Gooden e Bentum (2008) e Joshi e Aaron (2000) propuseram um modelo de múltiplos componentes, segundo o qual o desenvolvimento da leitura teria influência dos componentes cognitivos, de fatores ambientais (ecológicos) e psicológicos. O domínio ecológico inclui os componentes do ambiente doméstico e cultura, o envolvimento dos pais, o ambiente de sala de aula e o uso de dialetos, por exemplo. O fator escola, entendido como qualidade dos professores e/ ou métodos de ensino, tem impacto nas habilidades de leitura e escrita de crianças de séries iniciais de escolarização (Leybaert, Alégua, Deltour, \& Skinkel, 1997). Especialmente nas escolas públicas brasileiras, o professor precisa estar preparado para lidar com a diversidade de ambientes socioculturais e de conhecimentos de base das crianças (CarvaIho, 2000). Aliado a isso, salienta-se o desconhecimento de muitos professores em relação ao processo de aquisição da leitura e escrita (Freitas, 1989).
Da mesma forma, pais que tenham dificuldade em leitura e escrita podem ter mais dificuldade no auxílio à criança no que diz respeito às tarefas escolares, influenciando seu desempenho. Considerando que os pais podem ser mediadores em aprendizagens significativas para o desenvolvimento da criança, esta reflexão torna-se importante. Em relação ao neurodesenvolvimento infantil, Vygotsky já postulava que a internalização dos sistemas de signos altera o comportamento, sua percepção e interpretação do mundo. A interação com membros mais experientes é responsável pela incorporação de formas de comportamento estabelecidas. Sendo assim, quanto maior a experiência do indivíduo que lida com a criança, neste caso, os pais, maior a possibilidade dele agir como um facilitador do processo de aprendizagem que a criança terá do mundo. Assim, as funções mentais superiores tanto vão surgindo como se desenvolvendo. Então, sem o aporte sócio-histórico e cultural, o neurodesenvolvimento estaria comprometido ou não seria possível (Andrade \& Bueno, 2006). Se a experiência de leitura dos pais não é boa, com história pessoal de dificuldade escolar, vinculada a um baixo nível de escolaridade, poderá ser mais difícil funcionarem como mediadores e estimuladores do processo de aprendizagem de leitura e escrita de seus filhos. Portanto, é possível que, no grupo de crianças com dificuldade, os pais atuem menos como facilitadores do processo de aprendizagem.

Para Parente e Fonseca (2007), o estudo sobre a influência de fatores culturais na organização cerebral e no trabalho neuropsicológico de avaliação e de reabilitação decorre da noção de que existem diferenças individuais de funcionamento cerebral e de que estas diferenças podem ser genéticas ou resultado da interação entre cérebro, fatores ambientais e aprendizados adquiridos. Para as autoras, não há dúvida de que as múltiplas interações com o ambiente levam o cérebro a atuar de forma adaptativa. Sendo assim, os estímulos ambientais, a disponibilidade de jogos e materiais educacionais, a presença de livros e outros materiais de leitura, e a possibilidade de dispor de espaços próprios para a criança realizar as atividades e estudar potencializam as habilidades cognitivas e a aprendizagem (Moreno \& Cubero, 1995). Porém, a presença de recursos e materiais não é condição suficiente para garantir a aprendizagem da leitura e escrita. Há necessidade de interação com este material, o que inclui adultos com tempo e disposição para interagir com as crianças. Embora os pais tenham referido a presença de materiais e recursos, não há garantias de que exista efetiva interação das crianças com estes.

Segundo Marturano (2000), o interesse ativo dos pais pela criança, o investimento de tempo e recursos na sua criação e educação fornecem recursos emocionais essenciais ao desenvolvimento de um senso de competência, estimulando a autonomia e experiências sociais e culturais enriquecedoras. Ler para a criança, ouvir sua leitura, promover e compartilhar atividades de lazer, estimular a conversação, supervisionar o trabalho escolar, interessar-se pela escola, monitorar as companhias e amizades são atividades importantes para propiciar um desenvolvimento adequado 
das aprendizagens escolares. Esta autora afirma, ainda, que as expectativas e atribuições dos pais a respeito do sucesso da criança na escola podem afetar o rendimento de modo indireto, através de sua influência sobre o autoconceito. Moreno e Cubero (1995) confirmam que o autoconceito acadêmico está mais relacionado a percepções e expectativas dos pais com relação a eles do que com seu próprio rendimento acadêmico. O autoconceito mais positivo está vinculado à maior autonomia e competência das crianças sem dificuldade de aprendizagem (Okano, Loureiro, Linhares, \& Marturano, 2004).

O fato de os familiares do grupo com dificuldade deste estudo perceberem as dificuldades da criança na aprendizagem e no desempenho da leitura, e avaliarem o seu rendimento escolar como não adequado, pode demonstrar que, se os pais são sensíveis para perceber as dificuldades dos filhos, poderão ser sensíveis para ajudá-los ou para buscar recursos na tentativa de sua superação, aspecto fundamental no trabalho com crianças com dificuldade de aprendizagem. Por outro lado, a percepção dos familiares sobre o baixo desempenho escolar dos filhos pode levar os pais a terem uma menor expectativa sobre a aprendizagem dos mesmos e, assim, influenciar negativamente seu autoconceito e rendimento escolar. O impacto é ainda maior nos casos em que a criança tem história de reprovação.

No grupo competente, embora a diferença para com o grupo com dificuldade não seja significativa, aparecem mais relatos de que as crianças fazem as tarefas sozinhas, têm horários e local definido para as tarefas e há um tempo um pouco maior de contato da criança com o pai. Esses dados são indicativos de que estas crianças podem estar construindo um processo mais autônomo e organizado com relação à aprendizagem da leitura e escrita, e que talvez a família esteja conseguindo dar um suporte maior, com um ambiente mais estruturado.

Um estudo envolvendo crianças com queixas escolares, de D'Avila-Bacarji, Marturano e Elias (2005), que avaliou o suporte parental nos domínios acadêmico, desenvolvimental e emocional, apontou que as mães dessas crianças relataram menos suporte desenvolvimental e emocional, com problemas nas práticas educativas e relacionamento pais-criança conflituoso. Nos grupos pesquisados, com relação às práticas educativas parentais, aparece, no grupo com dificuldade, uma maior utilização da punição como forma de resolução de problemas, incluindo ameaças, castigo e força física. Já no grupo competente, o diálogo aparece com maior frequência como forma de resolução de conflitos. A punição está vinculada às técnicas coercitivas que têm por objetivo forçar a criança a se comportar da forma que os pais desejam, sendo que muitas famílias utilizam predominantemente esta prática no processo de educação dos filhos, mas tanto a punição física quanto a verbal tendem a ser prejudiciais ao desenvolvimento e integração familiar (Pacheco, Reppold, \& Hutz, 2005).

Outro fator a ser considerado são as mudanças familiares. Sabe-se que qualquer mudança no ambiente, que normalmente induz a um alto grau de tensão, qualquer evento de vida que pode ser considerado estressante, interfere nos padrões normais de resposta do indivíduo (Masten \& Garmezy, 1985). Observou-se, neste estudo, um maior número de mudanças familiares no grupo com dificuldade, sendo que, entre as mudanças mais citadas pelos participantes deste grupo, estão a morte de um familiar e a separação dos pais, situações estas que geram clima de tensão e estresse na família.

Não houve associação significativa entre os grupos nas variáveis referentes ao desenvolvimento infantil, sendo que esta dimensão, neste estudo, não está diretamente vinculada à habilidade de leitura e escrita das crianças. Porém, um aspecto interessante é que, considerando que as crianças do grupo com dificuldade foram avaliadas na perspectiva da neuropsicologia cognitiva e identificadas como crianças com dificuldade de aprendizagem na leitura e escrita, a grande maioria não recebe nenhum tratamento ou ajuda mais específica. A ausência de uma intervenção, na maioria dos casos, pode estar relacionada à dificuldade dos professores em diagnosticar problemas de escrita e leitura em crianças neste período, e à tendência destes em identificarem mais precocemente problemas de comportamento do que dificuldades de aprendizagem. Outro aspecto refere-se ao processo de "normalização" da dificuldade de leitura e escrita, reforçado pela presença de história familiar de dificuldade, levando à crença de que naquela família é natural ter dificuldades. Ainda é importante destacar a ausência de serviços especializados para avaliação e intervenção nas dificuldades de aprendizagem escolar disponíveis ou suficientes para atender a população de baixa renda, o que acarreta perda de um tempo importante de possibilidade de estimulação para o seu desenvolvimento.

Enfim, pesquisar os fatores psicossociais familiares na relação com a aprendizagem da leitura e escrita pode ajudar a identificar alguns fatores de risco e proteção para o desenvolvimento dessas habilidades nas crianças. Fatores que, no entanto, não devem ser interpretados como únicos responsáveis pelo desempenho escolar das crianças. Outros estudos já demonstraram os riscos de interpretações parciais de um fenômeno tão complexo, problematizando a inserção das camadas populares no universo escolar e a tendência de os professores evocarem a "demissão" ou "omissão" dos pais como causa dos problemas de aprendizagem (Charlot, 1997; Lahire, 1997), o que exoneraria a escola da necessidade de qualificar o processo de ensino e aprendizagem.

As constatações deste estudo de forma alguma devem reforçar a tese equivocada de que as famílias, especialmente as famílias pobres, são as responsáveis pelas dificuldades no desempenho escolar das crianças. Ao contrário, pretende-se que os dados sirvam para fortalecer políticas públicas de qualificação do processo educativo e de cuidado e atendimento às populações em situação de vulnerabilidade social e ambiental. Portanto, fica evidente a necessidade de uma rede de relacionamentos que funcione em prol do desenvolvimento das crianças e de suas famílias. 


\section{Considerações finais}

As habilidades de leitura e escrita envolvem múltiplos mecanismos perceptuais e cognitivos, identificados pela variabilidade de funções neuropsicológicas presentes no desempenho das crianças nessas atividades, mesmo quando têm dificuldades (Salles, 2008). Embora tenham sido encontrados alguns padrões relacionados às habilidades de consciência fonológica, memória fonológica e linguagem oral em estudos com crianças com dificuldade na leitura e escrita (Salles \& Parente, 2006b), observa-se grande variabilidade de perfis nas funções neuropsicológicas. Esta variabilidade nem sempre tem como fatores subjacentes os déficits em habilidades de processamento fonológico, como a maioria dos teóricos na área concorda em ser um fator responsável pelas dificuldades de leitura e escrita.

Nesse sentido, a neuropsicologia transcultural pode melhorar sua compreensão e abrangência na medida em que o sintoma passa a estar inserido dentro da circularidade de outros sistemas: emocional, familiar e psicossocial. Dessa forma, tal abordagem neuropsicológica permitirá compreender as várias formas dinâmicas em que sintomas e ambiente se articulam, rompendo com a possibilidade de uma análise biologicista do fenômeno das dificuldades da leitura e escrita, abrindo espaço para discussão dos fenômenos psicossociais que, neste estudo, referiram-se aos fatores familiares.

Ainda que o instrumento utilizado não objetivasse uma cobertura exaustiva dos recursos familiares relacionados ao sucesso ou insucesso na leitura e escrita, o mesmo ajudou a identificar algumas áreas mais representativas. Sendo assim, os resultados podem contribuir para fundamentar e direcionar intervenções neuropsicológicas e psicoeducativas, além de representar um desafio ao trabalho dos educadores na busca de uma maior integração com a família e na garantia de um processo de escolarização estimulador e de qualidade. Considerando que a família pode ser suporte à criança, é também com seus membros que o profissional da neuropsicologia pode estabelecer parcerias, no sentido de mobilização de recursos para o enfrentamento e prevenção dos problemas, sem, entretanto, exonerar a escola do processo educativo.

Pensar a dificuldade de leitura e escrita nesta dimensão mais ampliada, envolvendo fatores psicossociais familiares, permite propor uma avaliação diagnóstica neuropsicológica mais precisa e um planejamento interventivo mais eficiente.

Mesmo que este estudo tenha sido conduzido com uma amostra pequena de participantes, os resultados confirmam, claramente, a importância de se considerar os fatores psicossociais familiares na avaliação e compreensão das dificuldades de escrita e leitura em crianças. Deve-se ressaltar, também, que alguns dos resultados deste estudo são apenas tendências, sem associação significativa. Portanto, conclusões finais sobre a direção causal da relação entre esses fatores e desempenho em leitura e escrita não podem ser estabelecidas, sendo necessárias pesquisas mais am- plas, que incluam um maior número de crianças/famílias e a análise de outras variáveis, como as pedagógicas e escolares, para monitorar melhor a influência de tais fatores sobre o desempenho nas habilidades de ler e escrever.

\section{Referências}

Aaron, P. G. (1995). Diagnosis of Reading Disabilities. School Psychology Review, 24(3), 45-60.

Aaron, P. G., Joshi, R. M., Gooden, R., \& Bentum, K. E. (2008). Diagnosis and Treatment of Reading Disabilities Based on the Component Model of Reading. Journal of Learning Disabilities, 41(1), 67-84.

Angelini, A. L., Alves, I. C. B., Custódio, E. M., Duarte, W. F. \& Duarte, J. L. M. (1999). Matrizes Progressivas Coloridas de RAVEN escala especial. São Paulo: Centro Editor de Testes e Pesquisas em Psicologia.

Andrade, V. M., \& Bueno, O. F. A. (2006). A influência dos fatores socioculturais no neurodesenvolvimento. Em C. B. Mello, M. C. Miranda \& M. Muszkat, Neuropsicologia do desenvolvimento. São Paulo: Memnon.

Beitchman, J. H., \& Young, A. R. (1997). Learning Disorders with a Special Emphasis on Reading Disorders: A Review of the Past 10 Years. Journal of American Academy of Child and Adolescent Psychiatry, 36(8), 1020-1032.

Cardoso-Martins, C. (2008). Desenvolvimento das habilidades de leitura e escrita. Em D. Fuentes e cols., Neuropsicologia: teoria e prática. Porto Alegre: Artmed.

Carvalho, A. M. P. (2000). Baixo rendimento escolar: uma visão a partir do professor. Em C. A. R. Funayama (Org.), Problemas de aprendizagem: enfoque multidisciplinar (pp. 115-146). Campinas, SP: Alínea.

Charlot, B. (1997). Pour le savoir, contre la stratégie. Em F. Dubet (Org), École, famille : le malentendu (pp. 59-78). Paris: Éditions Textuel.

Charlot, B. (2000). Da relação com o saber. Elementos para uma teoria. Porto Alegre: Artmed.

D'Avila-Bacarji, K. M. G., Marturano, E. M., \& Elias, L. C. S. (2005). Suporte parental: um estudo sobre crianças com queixas escolares. Psicologia em Estudo, 10(1), 107-115.

DSM IV-TR. (2002). Manual diagnóstico e estatístico de transtornos mentais. Porto Alegre: Artes Médicas.

Ellis, A. W. (1995). Leitura, Escrita e Dislexia: uma análise cognitiva. Porto Alegre: Artes Médicas. 
Ellis, A. W., \& Young, A. W. (1988). Human Cognitive Neuropsychology. London: Lawrence Erlbaum Associates.

Freitas, L. (1989). A produção de ignorância na escola: uma análise crítica do ensino da língua escrita na sala de aula. São Paulo: Cortez.

Frith, U. (1997). Brain, mind and behaviour in dyslexia. Em C. Hulme \& M. Snowling (Org.), Dyslexia: biology, cognition and intervention (pp. 1-19). Singular Publishing Group, INC: San Diego.

Hillis, A. E., \& Caramazza, A. (1992). The Reading Process and Its Disorders. Em D. I. Margolin (Org), Cognitive Neuropsychology in Clinical Practice (pp.229-261). New York, Oxford Universitu Press.

Hutz, C., Koller, S., \& Bandeira, D. (1996). Resiliência e vulnerabilidade em crianças em situação de risco. Coletâneas da ANPEPP, 1, 7986.

IBGE - Instituto Brasileiro de Geografia e Estatística. Síntese dos Indicadores Sociais 2008. A Educação melhora mas ainda apresenta desafios. Recuperado: 08 nov 2011. Disponível: http:// www.ibge.gov.br/home/presidencia/noticias/noticia_visualiza. php?id_noticia=1233\&id_pagina=1.

Jong, P. F. (1998). Working Memory Deficits of Reading Disabled Children. Journal of Experimental Child Psychology, 70, 75-96.

Joshi, R. M., \& Aaron, P. G. (2000). The component model of reading: simple view of reading made a little more complex. Reading Psychology, 21, 85-97.

Lahire, B. (1997) Sucesso escolar nos meios populares: as razões do improvável. (R. A. Vasques \& S. Goldefer, Trad.). São Paulo: Ática.

Leybaert, J., Alégria, J., Deltour, J., \& Skinkel, R. (1997). Aprender a ler: o papel da linguagem, da consciência fonológica e da escola. Em J. Grégoire \& B. Piérart (Orgs.), Avaliação dos problemas de leitura: os novos modelos teóricos e suas implicações diagnósticas (pp. 143-166). Porto Alegre: Artes Médicas.

Marturano, E. M. (2000). Ambiente familiar e aprendizagem escolar. Em C. A. R. Funayama, Problemas de aprendizagem: enfoque muldisciplinar. Campinas SP: Alínea.

Marturano, E. M. (2006). O inventário de recursos do ambiente familiar. Psicologia: Reflexão e Crítica, 19(3), 498-506.

Mayringer, H., \& Wimmer, H. (2000). Pseudoname Learning by German-Speaking Children with Dyslexia: Evidence for a Phonological Learning Deficit. Journal of Experimental Child Psychology, 75, 116-133.

Masten, A. S., \& Garmezy, N. (1985). Risk, vulnerability, and protective factors in developmental psychopathology. Em B. B. Lahey \& A. E. Kazdin (Eds.), Advances in Clinical Child Psychology (pp.1-52). New York: Plenum Press.
Miranda, M. C., \& Muszkat, M. (2004). Neuropsicologia do desenvolvimento. Em V. M. Andrade, F. H. Santos \& F. A. B. Orlando, Neuropsicologia hoje. São Paulo: Artes Médicas.

Morais, J. (1996). A arte de ler. São Paulo: Editora da Universidade Estadual Paulista.

Moreno, M. C., \& Cubero, R. (1995). Relações sociais nos anos préescolares: família, escola, colegas. Em C. Col, J. Palácios \& A. Marchesi (Orgs.), Desenvolvimento psicológico e Educação Psicologia Evolutiva (pp. 190-202). Porto Alegre: Artes Médicas.

Nachmias, C. F., \& Nachmias, D. (1996). Research methods in the social sciences. London: Martin's Press.

Okano, C. B., Loureiro, S. R., Linhares, M. B. M., \& Marturano, E. M. (2004). Crianças com dificuldades escolares atendidas em programa de suporte psicopedagógico na escola: avaliação de autoconceito. Psicologia: Reflexão e Crítica, 17(1), pp. 121-128.

Parente, M. A. M. P., \& Fonseca, R. P. (2007). A importância de fatores culturais nos estudos neuropsicológicos. Em E. C. Macedo, L. I. Z. Mendonça, B. B. G Schlecht, K.Z Ortiz \& D. A. Azambuja (Orgs.), Avanços em neuropsicologia: das pesquisas à aplicação clínica. São Paulo: Santos Editora.

Pacheco, J., Reppold, C. T., \& Hutz, C. S. (2005). Modelos de intervenção parental para tratamento de crianças e adolescentes com problemas de comportamento. Em C. S. Hutz (Org.), Violência e risco na infância e adolescência: pesquisa e intervenção. São Paulo: Casa do Psicólogo.

Pennington, B. F. (1997). Diagnóstico de Distúrbios de Aprendizagem: um referencial neuropsicológico. São Paulo: Pioneira.

Salles, J. F. (2005) Habilidades e dificuldades de leitura e escrita em crianças de $2^{a}$ série: abordagem neuropsicológica cognitiva. Tese de Doutorado, Programa de Pós-Graduação em Psicologia do Desenvolvimento, Universidade Federal do Rio Grande do Sul, Porto Alegre, Rio Grande do Sul.

Salles, J. F., \& Parente, M. A. P. (2006a). Heterogeneidade nas estratégias de leitura/escrita em crianças com dificuldade de leitura/escrita. Revista Psico, 37(1), pp. 83-90.

Salles, J. F., \& Parente, M. A. P. (2006b). Funções neuropsicológicas em crianças com dificuldades de leitura e escrita. Psicologia: Teoria e Pesquisa, 22(2), 153-162.

Salles, J. F., \& Parente, M. A. P. (2007). Avaliação da leitura e escrita de palavras em crianças de $2^{\text {a }}$ série: abordagem neuropsicológica cognitiva. Psicologia Reflexão e Crítica, 20(2), 218-226.

Salles, J. F., \& Parente, M. A. P. (2008). Variabilidade no desempenho em tarefas neuropsicológicas entre crianças de $2^{\text {a }}$ série com dificuldades de leitura e escrita. Arquivos Brasileiros de Psicologia, $60(1)$. 
Torgesen, J. K., Wagner, R. K., \& Rashotte, C. A. (1994). Longitudinal Studies of Phonological Processing and Reading. Journal of Learning Disabilities, 27(5), 276-286.
Wimmer, H. (1993). Characteristics of developmental dyslexia in a regular writing system. Applied Psycholinguistics, 14, 1-33.

Recebido em: 04/05/2009

Reformulações: 22/06/2010 (1a.)

$24 / 08 / 2011\left(2^{\mathrm{a}}\right)$

Aprovado em: 06/09/2011

\section{Sobre as autoras:}

Jacqueline Raquel Bianchi Enricone (jenricone@uri.com.br)

Prof. Ms. Curso de Psicologia, Universidade Regional Integrada do Alto Uruguai e das Missões, URI - Campus de Erechim.

Jerusa Fumagalli de Salles (jerusafs@yahoo.com.br / website: www.ufrgs.br / neuropsicologia)

Prof. Dra Instituto de Psicologia, Universidade Federal do Rio Grande do UFRGS, Programa de Pós-Graduação em Psicologia.

\section{Endereço para correspondência:}

Prof. Dra Jerusa Fumagalli de Salles: Av. Ramiro Barcelos, 2600, sala 114. Instituto de Psicologia - UFRGS. Porto Alegre - RS. CEP: $90035-003$ Fone: (51) 3308-5111.

\section{Endereço completo das autoras:}

Prof. Jacqueline Raquel Bianchi Enricone

Av. Sete de Setembro, 1621. URI - Campus de Erechim. Erechim/RS. CEP: 99700-000. Fone: (54) 3520-9000 Fax: (54) 3520-9090

Prof. Dr ${ }^{a}$ Jerusa Fumagalli de Salles

Av. Ramiro Barcelos, 2600, sala 114. Instituto de Psicologia - UFRGS. Porto Alegre - RS. CEP: 90035-003 Fone: (51) 3308-5111. 